\title{
Gut Microbiome Signatures of Risk and Prodromal Markers of Parkinson Disease
}

\author{
Sebastian Heinzel PhD $0,{ }^{1}$ Velma T. E. Aho PhD $0^{2,3}$ Ulrike Suenkel MD, ${ }^{4}$ \\ Anna-Katharina von Thaler $\mathrm{PhD},{ }^{4}$ Claudia Schulte MSc, ${ }^{4,5}$ Christian Deuschle, ${ }^{4,5}$ \\ Lars Paulin MSc, ${ }^{2}$ Sari Hantunen $\mathrm{PhD}^{6}{ }^{6}$ Kathrin Brockmann MD, ${ }^{4,5}$ \\ Gerhard W. Eschweiler MD, ${ }^{7,8}$ Walter Maetzler MD, ${ }^{1}$ Daniela Berg MD, ${ }^{1,4}$ \\ Petri Auvinen $\mathrm{PhD}^{2}$ and Filip Scheperjans MD $\mathbb{1}^{3}$
}

\begin{abstract}
Objective: Alterations of the gut microbiome in Parkinson disease (PD) have been repeatedly demonstrated. However, little is known about whether such alterations precede disease onset and how they relate to risk and prodromal markers of PD. We investigated associations of these features with gut microbiome composition.

Methods: Established risk and prodromal markers of PD as well as factors related to diet/lifestyle, bowel function, and medication were studied in relation to bacterial $\alpha$-/ $\beta$-diversity, enterotypes, and differential abundance in stool samples of 666 elderly TREND (Tübingen Evaluation of Risk Factors for Early Detection of Neurodegeneration) study participants.

Results: Among risk and prodromal markers, physical activity, occupational solvent exposure, and constipation showed associations with $\alpha$-diversity. Physical activity, sex, constipation, possible rapid eye movement sleep behavior disorder (RBD), and smoking were associated with $\beta$-diversity. Subthreshold parkinsonism and physical activity showed an interaction effect. Among other factors, age and urate-lowering medication were associated with $\alpha$ - and $\beta$-diversity. Physical inactivity and constipation were highest in individuals with the Firmicutes-enriched enterotype. Constipation was lowest and subthreshold parkinsonism least frequent in individuals with the Prevotella-enriched enterotype. Differentially abundant taxa were linked to constipation, physical activity, possible RBD, smoking, and subthreshold parkinsonism. Substantia nigra hyperechogenicity, olfactory loss, depression, orthostatic hypotension, urinary/erectile dysfunction, PD family history, and the prodromal PD probability showed no significant microbiome associations.

Interpretation: Several risk and prodromal markers of PD are associated with gut microbiome composition. However, the impact of the gut microbiome on PD risk and potential microbiome-dependent subtypes in the prodrome of PD need further investigation based on prospective clinical and (multi)omics data in incident PD cases.
\end{abstract}

ANN NEUROL 2020;88:320-331

$T^{\text {he }}$

he presence of gastrointestinal pathological $\alpha$-synuclein deposits and constipation in prodromal and clinically established Parkinson disease (PD) suggests an integral role of the gut-brain axis for the early pathogenesis of PD. ${ }^{1-3}$ The synucleinopathy is hypothesized to ascend via the vagal nerve from peripheral neurons of the

View this article online at wileyonlinelibrary.com. DOI: 10.1002/ana.25788

Received Dec 11, 2019, and in revised form May 19, 2020. Accepted for publication May 19, 2020

Address correspondence to Dr Heinzel, Department of Neurology, Christian-Albrecht University of Kiel, Arnold-Heller-Straße 3, 24105 Kiel, Germany. E-mail: s.heinzel@neurologie.uni-kiel.de

From the ${ }^{1}$ Department of Neurology, Christian-Albrechts University of Kiel, Kiel, Germany; ${ }^{2}$ Institute of Biotechnology, University of Helsinki, Helsinki, Finland; ${ }^{3}$ Department of Neurology, Helsinki University Hospital and Department of Clinical Neurosciences (Neurology), University of Helsinki, Helsinki, Finland; ${ }^{4}$ Department of Neurodegeneration, Hertie Institute for Clinical Brain Research, University of Tübingen, Tübingen, Germany; ${ }^{5}$ German Center for Neurodegenerative Diseases, University of Tübingen, Tübingen, Germany; ${ }^{6}$ Institute of Public Health and Clinical Nutrition, University of Eastern Finland,

Kuopio, Finland; ${ }^{7}$ Department of Psychiatry and Psychotherapy, University of Tübingen, Tübingen, Germany; and ${ }^{8}$ Geriatric Center at the University Hospital Tübingen, Tübingen, Germany

Additional supporting information can be found in the online version of this article.

320 (C) 2020 The Authors. Annals of Neurology published by Wiley Periodicals, Inc. on behalf of American Neurological Association.

This is an open access article under the terms of the Creative Commons Attribution-NonCommercial-NoDerivs License, which permits use and distribution in any medium, provided the original work is properly cited, the use is non-commercial and no modifications or adaptations are made. 
gastrointestinal tract to the brain. ${ }^{4}$ Moreover, increased intestinal permeability, ${ }^{5}$ elevated stool inflammatory cytokines, ${ }^{6}$ and colonic wall inflammation ${ }^{7}$ have been shown in PD patients, and may also represent key gastrointestinal processes in prodromal PD. Mice overexpressing $\alpha$-synuclein show aggravated motor dysfunction when colonized with intestinal microbiota from PD patients. ${ }^{8}$ However, the specific role of gut microbiota for PD and the factors modulating such processes along the microbiota-gutbrain axis are still largely unknown. The most consistently shown PD-related changes of gut microbial composition include an increase in the relative abundances of Verrucomicrobiaceae and Akkermansia and a decrease in Prevotellaceae and Prevotella. ${ }^{9,10}$ The latter has also been associated with progressive PD motor symptoms over 2 years ${ }^{11}$ and with rapid eye movement sleep behavior disorder (RBD), ${ }^{12}$ a highly specific prodromal marker of PD. Prospective evidence of several PD risk markers (indicating an increased PD risk) and prodromal markers (indicating an already initiated neurodegenerative process) confirm the concept of a prodromal phase preceding clinically established PD by years or even decades. ${ }^{13}$ Based on this evidence, it is possible to define specific predictive values for these markers and to calculate individual prodromal PD probabilities based on age and marker profiles. ${ }^{14,15}$ $\mathrm{Age}^{16}$ and several markers established in the International Parkinson and Movement Disorder Society (MDS) research criteria for prodromal $\mathrm{PD}^{14,15}$ have also been associated with gut microbial changes; these include risk markers such as male sex, ${ }^{17}$ diabetes, ${ }^{18}$ nonsmoking, and nonuse of caffeine ${ }^{16,17}$ and prodromal markers, such as $\mathrm{RBD},{ }^{12}$ constipation, ${ }^{16}$ and depression. ${ }^{19}$ Moreover, physical inactivity, a risk marker for $\mathrm{PD}^{15}$ and for most major chronic diseases occurring frequently in old age, ${ }^{20}$ has been reported to affect microbial $\beta$-diversity in elderly males ${ }^{21}$ as well as various inflammatory and immune processes. ${ }^{22}$ However, these studies focused on microbial associations with single or few PD-related markers, or studied very small samples considering the multitude of other markers and confounding factors that may modulate the gut microbiome. For instance, factors related to diet, bowel function, and disease/medication often exert effects on microbial composition, ${ }^{16-18}$ and may thus bias findings of marker associations. The present study therefore assessed gut microbial diversity, enterotypes, and taxonomic composition and investigated their associations with a comprehensive set of PD risk and prodromal markers, the overall prodromal PD probability, and a wide range of other potential confounders in a large sample of elderly individuals.

\section{Patients and Methods}

All subjects were participants of the prospective Tübingen Evaluation of Risk Factors for Early Detection of Neurodegeneration (TREND) study. The cohort has been enriched regarding an increased PD risk by partly recruiting participants based on the presence of olfactory loss, depression, and/or possible RBD. Biennial comprehensive assessments in 1,202 individuals have been performed over the past 10 years (www.trend-studie.de/english). Stool samples were collected at the third follow-up of the study and associated with markers/factors assessed at the corresponding wave of assessments. Stool was sampled using collection tubes containing a DNA stabilizer (PSP Spin Stool DNA Plus Kit; STRATEC Molecular, Birkenfeld, Germany), provided using postal services and frozen and stored at $-80^{\circ} \mathrm{C}$ immediately upon arrival. Samples were available from 745 participants. After excluding individuals taking antibiotic medication $(\mathrm{n}=47)$, patients with $\mathrm{PD}(\mathrm{n}=11)$ or atypical/secondary parkinsonism $(n=2)$, incident cases of PD $(n=3)$, and individuals with missing dietary and medication data $(\mathrm{n}=16)$, data from 666 individuals were included in the analyses.

In total, 9 risk and 9 prodromal markers as defined by the recently updated MDS research criteria for prodromal PD were selected a priori and investigated for associations with microbial measures. ${ }^{15}$ We assessed the PD risk markers male sex, substantia nigra hyperechogenicity (transcranial ultrasound), nonsmoking, no consumption of coffee (cups per day), positive PD family history (first-degree relatives), physical activity (ie, low activity levels as measured using hours of sport/wk), type 2 diabetes (diagnosis), and occupational solvent and pesticide exposure (self-reported). Prodromal markers comprised olfactory loss (Sniffin' Sticks, 16 SS), depression (acute or lifetime diagnosis), constipation (Rome III criteria, sum score), ${ }^{23}$ possible RBD (RBD screening questionnaire), excessive daytime somnolence, erectile and urinary dysfunction, and symptomatic orthostatic hypotension (the latter 3 assessed using the Unified Multiple System Atrophy Rating Scale questionnaire as described previously). ${ }^{24}$ Moreover, motor deficits indicating subthreshold parkinsonism were assessed using the MDS Unified Parkinson's Disease Rating Scale part III ${ }^{25}$ (score $>6$ after excluding action/postural tremor items; scores from 3 to 6 were defined as borderline motor deficit). ${ }^{14}$ Furthermore, 90 additional variables were considered comprising:

- Eighty variables that were screened as potential confounders for adjustment in the statistical analyses (see statistical approach below) 
- Seven generic/physiological variables (eg, age, body mass index [BMI], education, and exhaustion from climbing 3 levels of stairs [no, yes, not capable])

- Five variables related to bowel function (eg, irritable bowel syndrome, bloating, vomiting/diarrhea)

- Sixteen diet-related variables (assessing weekly meat, vegetables, dairy, protein, carbohydrate, and alcohol consumption)

- One variable on gout

- Fifty-one variables related to medication

- Exploratory variables that measure further aspects of prodromal PD or represent alternative (yet less established) ${ }^{14}$ groupings/definitions of risk/prodromal markers

- Prodromal PD probability values, and diagnoses of possible ( $>50 \%$ probability) and probable prodromal PD $(>80 \%)$ as calculated based on age and comprehensive individual marker profiles according to the MDS research criteria for prodromal $\mathrm{PD}^{14}$

- Exploratory alternative groupings/definitions of risk/ prodromal markers comprised 3 variables on (functional) constipation, 2 variables on smoking status/ history, and 2 variables on motor ratings

For the complete list of the 18 risk and prodromal markers and the 90 additional variables, their specific assessment methods, variable definitions, and descriptive statistics, see the Supporting Material. The study was approved by the local ethical committee (Medical Faculty, University of Tübingen; 444/2019BO2). All participants provided written informed consent.

\section{Stool Sample DNA Extraction, Library Preparation, and Sequencing}

DNA extraction, library preparation, and sequencing were performed at the DNA Sequencing and Genomics Laboratory of the Institute of Biotechnology, University of Helsinki. All samples were stored at $-80^{\circ} \mathrm{C}$ and randomized in a $-20^{\circ} \mathrm{C}$ room before bulk DNA extraction with the PSP Spin Stool DNA Plus Kit (STRATEC Molecular). One blank was added per extraction batch to assess potential contamination. After extraction, DNA concentrations were measured with NanoDrop ND-1000 (Thermo Fisher Scientific, Waltham, MA). V3-V4 regions of the bacterial 16S ribosomal RNA gene were amplified in Verity 96-well Thermal Cyclers (Thermo Fisher Scientific) using a previously described polymerase chain reaction (PCR) protocol. ${ }^{11}$ The amount of template DNA used for the first PCR ranged between $11.25 \mathrm{ng}$ and $1311.26 \mathrm{ng}$. Each PCR batch included blank samples for assessment of potential contamination. Dual indexes were used in the second PCR; these had been selected using BARCOSEL ${ }^{26}$ to allow pooling and sequencing of all samples in 1 pool run on 3 separate runs on a MiSeq (Illumina, San Diego, CA; v3 600 cycle kit, forward/reverse read length 328/278 bases). Thus, each sample was sequenced 3 times among all the other samples, reducing possible batch effects.

\section{Sequence Analysis}

The raw sequence data contained 48,782,168 sequence reads (availability: ENA accession number PRJEB32920). We combined the sequence reads from the 3 sequencing runs, and then trimmed primers and low-quality sequences with cutadapt (v1.8.3, ${ }^{27}$ parameters $\mathrm{q}=30$ and $\mathrm{m}=160)$. Merging paired reads, alignment to a reference database (SILVA, v132), chimera removal, taxonomic classification (reference database: Ribosomal Database Project (RDP), v16, 16S rRNA reference (PDS)), and operational taxonomic unit (OTU) clustering ("cluster.split" approach) were run with mothur (v1.40.0), ${ }^{28}$ following the standard operating procedure (SOP) for MiSeq data. ${ }^{29}$ Parameters differing from the SOP were maxlength $=500$ and maxhomop $=8$ for the first "screen.seqs," start $=2$ and end $=17012$ for the second "screen.seqs," diffs $=4$ for "pre.cluster," cutoff = 70 for "classify.seqs," and keeping archaeal sequences in "remove.lineage." Additionally, singleton sequences were removed prior to "classify.seqs" using "split.abund" with cutoff $=1$. After excluding data from blanks and all OTUs with $\leq 10$ sequence reads, the final dataset consisted of $25,390,744$ sequence reads $(34,082 \pm 4,785$ per sample).

\section{Microbial Measures}

All microbiota analyses were performed using genus-level data. $\alpha$-Diversity was estimated with the inverse Simpson index ( $\mathrm{R}$ package: phyloseq). ${ }^{30}$ This index summarizes richness (number of different taxonomic units) and evenness (abundance distribution of taxonomic units) for each sample. The measure chosen for $\beta$-diversity was Bray-Curtis dissimilarity ( $\mathrm{R}$ package: vegan). It quantifies the intersample compositional dissimilarity based on both presence/absence of taxonomic units and their abundances. Nonmetric multidimensional scaling (NMDS; R package: vegan) was applied to produce an ordination based on rank orders in the BrayCurtis dissimilarity matrix, and to plot compositional dissimilarities between samples (and groups of samples) in a 2dimensional ordination space.

Microbial enterotypes ${ }^{31}$ were determined using the algorithm provided at http://enterotypes.org/. All unclassified taxa (genus level) were excluded from $\alpha$-diversity, enterotype, and differential abundance analyses. For differential abundance, analyses were also performed for the family level and OTU level. 


\section{Statistical Analyses}

The present study investigates possible associations of 18 single risk and prodromal markers with different microbial measures. To investigate this primary objective, potentially confounding effects of 80 different factors were considered for adjustment of risk and prodromal marker effects in the statistical analysis. Moreover, 10 variables measuring additional aspects of PD (eg, prodromal PD probabilities) or alternative marker groupings/definitions entered the analyses as exploratory variables (mentioned above and listed in the Supporting Material). The study used a 2-stage statistical analysis approach. First, in a prescreening step, each of the 18 risk and prodromal markers as well as each of the 90 additional variables were tested separately (for specific tests, see below) for associations with microbial measures ( $\alpha$-diversity, $\beta$-diversity, enterotypes). Here, markers/variables showing effects with $p<0.1$ were selected for subsequent multifactorial statistical modeling. From the multiple measures of constipation, smoking, and motor function, the variable with the lowest $p$ value was selected. Results of the prescreening step are shown in the Supporting Material. In a second step, all selected risk and prodromal markers and additional confounding adjustment factors were entered into multifactorial statistical models, and model selection was performed with final models only comprising markers/factors showing marginal effects with $p<0.1$ (bolded $p$ values in the Supporting Material: single variable association). Because the primary objective of the study was association testing for the 18 risk and prodromal markers (which were tested at least in the prescreening step), Bonferroni corrections for $\mathrm{n}=18$ tests were applied in the multifactorial tests, and effects of the 18 markers with a corrected threshold of $p<0.003$ were considered significant. For adjustment factors, no correction for multiple testing was applied $(p<0.05)$. In enterotype analyses, differences between all 3 enterotype groups were statistically tested post hoc pairwise, and accordingly effects with $p<0.0009$ were considered

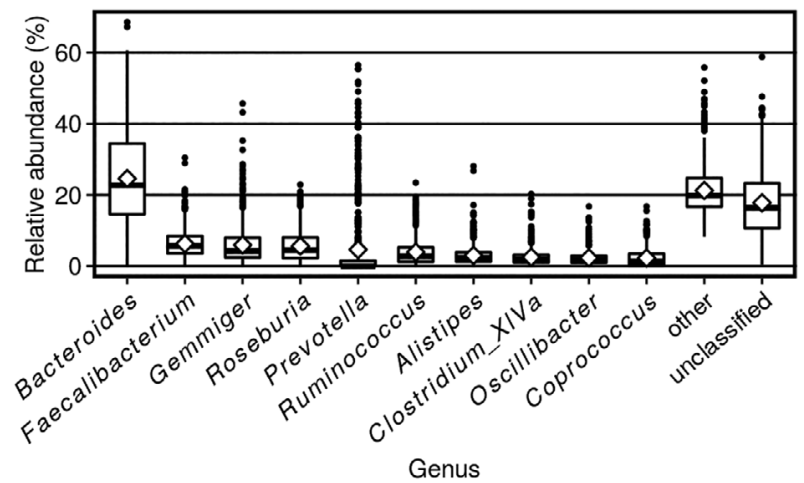

FIGURE 1: Relative abundances of the 10 most common bacterial genera. significant after correction for multiple testing for $\mathrm{n}=18 \times 3=54$ tests.

Linear multiple regressions of $\alpha$-diversity were performed. Associations of markers/factors with $\beta$-diversity were tested using PERMANOVAs (single variable: adonis, multifactorial: adonis2, both commands from the $\mathrm{R}$ package vegan). Moreover, $\alpha$ - and $\beta$-diversity were tested for interaction effects between physical activity and other risk and prodromal markers of PD. Marker/factor differences between enterotypes were tested using Fisher exact tests and Kruskal-Wallis tests, and subsequent multifactorial analyses using multinomial logistic regressions with enterotype as dependent variable.

For differential abundance analysis, we used DESeq2, a method based on generalized linear models with negative binomial distributions (sequence count data). The DESeq2 model included all covariates accounted for in the final PERMANOVA model for $\beta$-diversity, and $p$ values were adjusted for multiple testing using false discovery rate (FDR) corrections accounting for the number of different taxa tested. We used $\mathrm{R}$ (v3.5.1) for all analyses and figures. TREND study data were collected and managed using REDCap electronic data capture tools hosted at the University of Tübingen.

\section{Results}

\section{Descriptive Statistics}

The TREND study sample $(\mathrm{n}=666)$ had a mean age $( \pm \mathrm{SD}$, range) of $68.4 \pm 6.3$ years (53-86). Risk and prodromal marker variables showed the following descriptive statistics: male sex (52.7\%), substantia nigra hyperechogenicity (21.6\%), positive PD family history (14.7\%), physical inactivity (no activity, 25.7\%; <1 h/wk, 30.9\%; $1-2 \mathrm{~h} / \mathrm{wk}$, $24.2 \%$; $2-4 \mathrm{~h} / \mathrm{wk}, 8.3 \%$; $>4 \mathrm{~h} / \mathrm{wk}, 10.4 \%)$, nonsmoking (never, 6.8\%; former, 46.7\%; current smoker, 46.5\%), nonuse of coffee $(14.4 \%)$, type 2 diabetes $(8.3 \%)$, occupational solvent exposure $(10.8 \%)$, occupational pesticide exposure (1.3\%), olfactory loss (19.7\%), depression (31.5\%), constipation (severity sum score $=3.1 \pm 3.9$, range $=0-25)$, possible $\operatorname{RBD}(12.6 \%)$, excessive daytime somnolence (3.8\%), erectile dysfunction (in males, $21.5 \%$ ), urinary dysfunction (5.1\%), symptomatic orthostatic hypotension $(4.7 \%)$, and regarding motor functions, no motor deficit $(90.5 \%)$, borderline motor deficit $(6.0 \%)$, and subthreshold parkinsonism (3.5\%). Based on prodromal PD probabilities $(3.8 \pm 11.4 \%, \quad 0.0-89.1 \%), \quad 15$ individuals (2.3\%) were diagnosed with possible prodromal PD and 5 individuals $(0.8 \%)$ with probable prodromal PD. The most abundant bacterial genus in the subjects' stool samples was Bacteroides, followed by Faecalibacterium, Gemmiger, Roseburia, Prevotella, and Ruminococcus (Fig 1). 


\begin{tabular}{|c|c|c|c|}
\hline & Estimate & SE & $p$ \\
\hline Intercept & 4.05 & 1.24 & $0.00113^{\mathrm{a}}$ \\
\hline \multicolumn{4}{|l|}{ Risk markers } \\
\hline Physical activity (h sport/wk) & -0.23 & 0.09 & $0.00765^{\mathrm{b}}$ \\
\hline Occupational solvent exposure (no, yes) & -0.91 & 0.32 & $0.00401^{\mathrm{b}}$ \\
\hline \multicolumn{4}{|l|}{ Prodromal markers } \\
\hline $\begin{array}{l}\text { Constipation (number of fulfilled Rome III criteria } \\
\text { items of functional constipation) }\end{array}$ & 0.23 & 0.12 & $0.04540^{\mathrm{b}}$ \\
\hline Subthreshold parkinsonism (no, borderline, yes) & 1.06 & 0.56 & 0.05930 \\
\hline Physical activity $\times$ subthreshold parkinsonism & -0.33 & 0.19 & 0.08769 \\
\hline \multicolumn{4}{|l|}{ Additional adjustment factors } \\
\hline Age (yr) & 0.03 & 0.02 & $0.04757^{b}$ \\
\hline Exhaustion from climbing stairs (no, yes, not capable) & -0.75 & 0.21 & $0.00038^{\mathrm{b}}$ \\
\hline Urate-lowering medication (no, yes) & -1.25 & 0.47 & $0.00812^{\mathrm{b}}$ \\
\hline Thyroid medication (no, yes) & -0.61 & 0.24 & $0.00951^{\mathrm{b}}$ \\
\hline Total dairy consumption (score) & -0.20 & 0.11 & 0.07369 \\
\hline
\end{tabular}

\section{$\alpha$-Diversity}

$\alpha$-Diversity on the genus level as indicated by the inverse Simpson index showed associations $(p<0.05)$ with 2 risk markers, 1 prodromal marker, and 4 additional adjustment factors (Table 1). Overall, the multiple regression model explained $8.0 \%$ of $\alpha$-diversity variance (adjusted $R^{2}$ ). Constipation severity and age were positively associated with $\alpha$-diversity, whereas physical activity and occupational solvent exposure as well as intake of thyroid medication, urate-lowering medication, and exhaustion from walking stairs were inversely associated with $\alpha$-diversity (Fig 2). None of the selected variables showed an interaction $(p>0.1)$ with physical activity on $\alpha$-diversity. When entering motor deficits into the regression, no association with $\alpha$-diversity was observed $(p>0.1)$, and the interaction of motor deficits and physical activity was nonsignificant $(p=0.088)$.

\section{$\beta$-Diversity}

Intersample differences in microbial composition as indicated by $\beta$-diversity showed significant associations with multiple risk and prodromal markers of PD (Table 2). In addition to age, physical activity, constipation, and BMI explained most of the variance $\left(R^{2}\right.$; Fig 3$)$. Moreover, sex, smoking, possible RBD, different medications, and dark bread consumption showed associations with $\beta$-diversity. Although motor deficits showed no effect, the interaction between physical activity and motor deficits explained variance in $\beta$-diversity $(p=0.002)$.

\section{Enterotypes}

Bacteroides-enriched $(70.9 \%$ of samples) microbiomes were more frequent compared to Prevotella-enriched (21.5\%) and Firmicutes-enriched (7.7\%) microbiomes. Enterotypes differed in several risk and prodromal markers and additional factors (Table 3). For instance, the lowest physical activity levels as well as most severe constipation were observed for the Firmicutes-enriched enterotype (Fig 4). Higher physical activity levels were linked to the Bacteroides-enriched enterotype. Constipation severity was lowest and subthreshold parkinsonism least frequently observed in individuals with the Prevotella-enriched enterotype. 


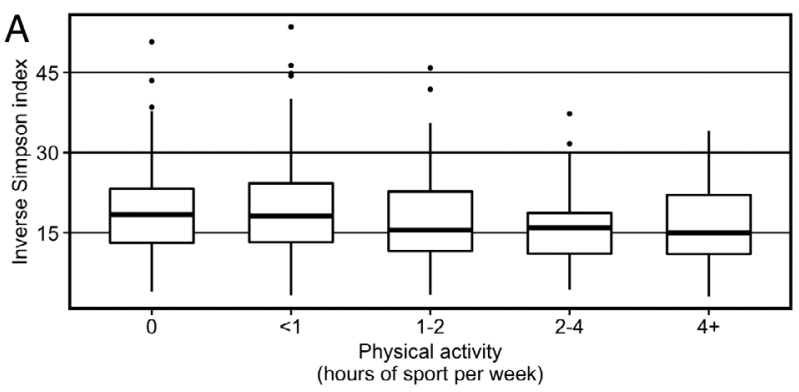

C

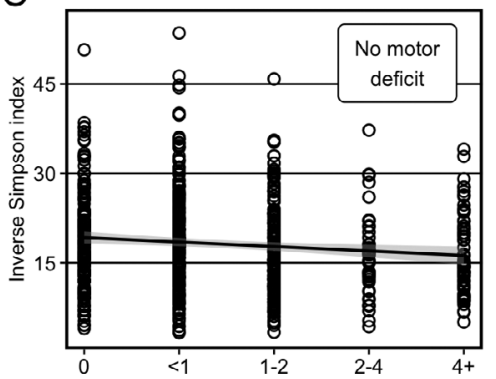

B

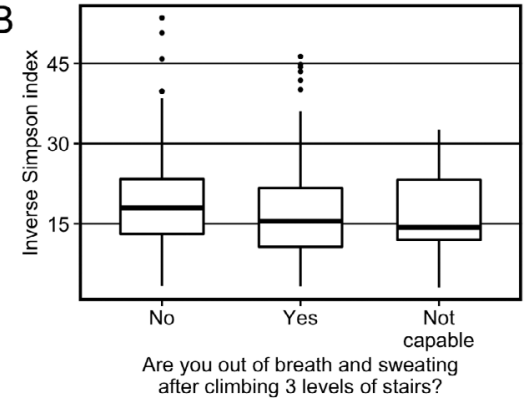

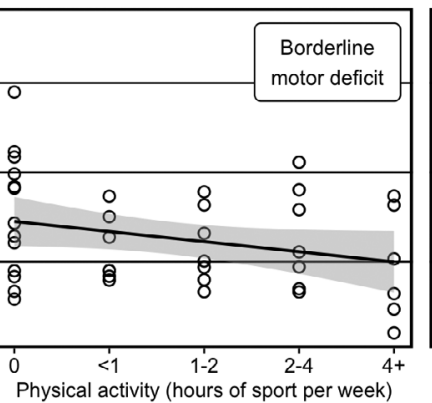

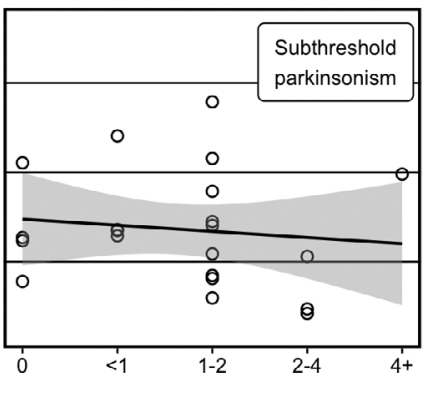

FIGURE 2: $\alpha$-Diversity and physical motor measures. Magnitude of $\alpha$-diversity by (A) levels of weekly physical activity, (B) physical exhaustion, and (C) physical activity levels in different motor deficit groups.

TABLE 2. Associations of $\beta$-Diversity with Risk and Prodromal Markers in Parkinson Disease and Additional Factors$$
R^{2}
$$$$
\boldsymbol{F}
$$$$
p
$$

Risk markers

Physical activity (h sport/wk)

Male sex (no, yes)

Smoking (pack-years)

Prodromal markers

Constipation (sum score of Rome III criteria

items of functional constipation)

Possible RBD (no/yes)

Subthreshold parkinsonism (no, borderline, yes)

Physical activity $\times$ subthreshold parkinsonism

Additional adjustment factors

Age (yr)

Diabetes medication (no, yes)

Urate-lowering medication (no, yes)

Beta-blocker medication (no, yes)

Dark bread (no, yes)

BMI $\left(\mathrm{kg} / \mathrm{m}^{2}\right)$
0.014

0.006

0.004

0.009

0.003

0.002

0.006

0.008

4.88

2.66

2.43

1.91

2.19

8.41

2.36

5.48

0.004

2.14

0.004

0.003

0.003

0.013 $0.001^{\mathrm{a}}$

$0.003^{\mathrm{a}}$

$0.020^{\mathrm{b}}$

$0.002^{\mathrm{a}}$

$0.037^{\mathrm{b}}$
1.45

0.159

3.56

$0.002^{\mathrm{a}}$

${ }^{\text {a }}$ ignificant effects of risk and prodromal markers in permutational multivariate analysis of variance models after Bonferroni correction for multiple testing $(p<0.003)$.

${ }^{\mathrm{b}}$ Effects with an uncorrected $p<0.05$.

$\mathrm{BMI}=$ body mass index; $\mathrm{RBD}=$ rapid eye movement sleep behavior disorder. 

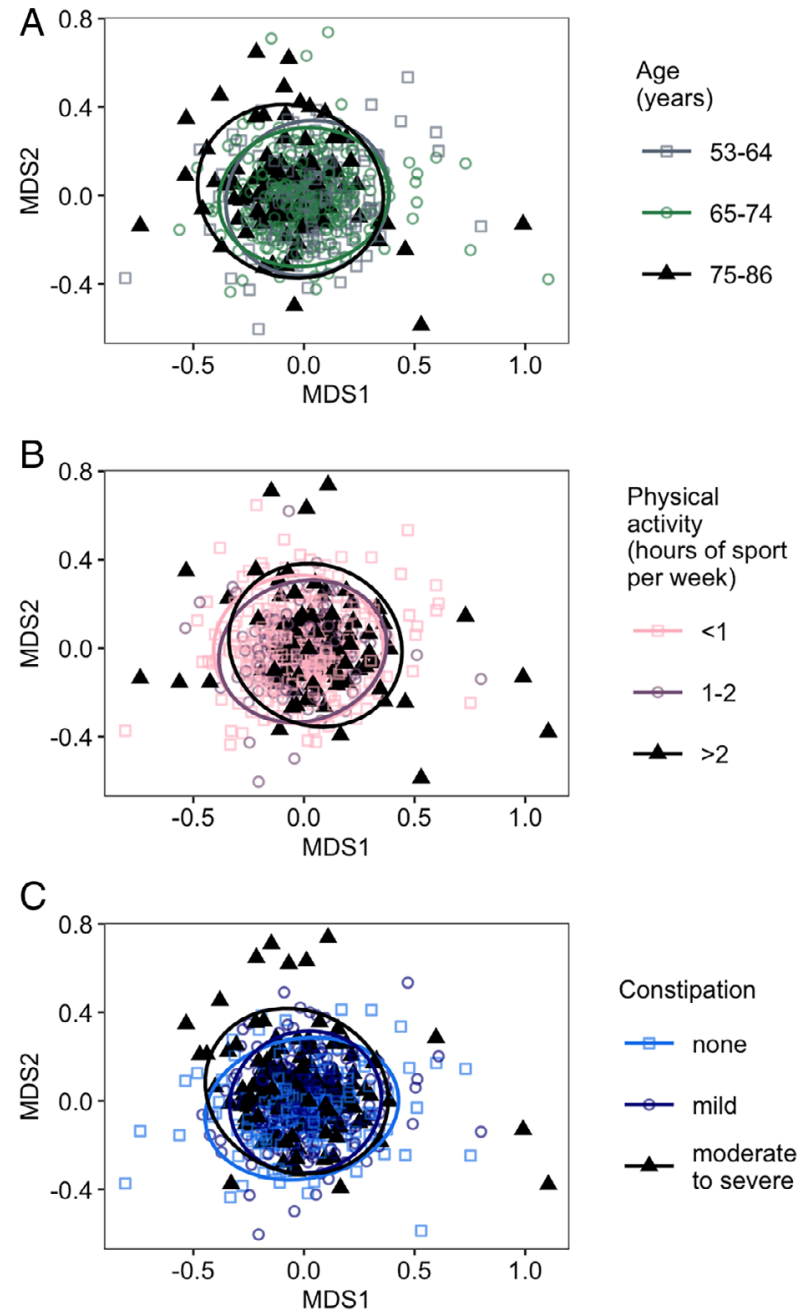

FIGURE 3: Nonmetric multidimensional scaling (NMDS) plots in 2-dimensional space (MDS1 and 2) of factors showing significant Bray-Curtis distance differences. Differences in microbial composition between (A) age groups, (B) groups with different physical activity levels, and (C) groups stratified by severity of constipation are shown. Circles indicate $95 \%$ confidence intervals.

\section{Differential Abundance}

Several of the variables associated with $\beta$-diversity were also linked to the abundances of specific taxa (Supporting Material; all $p$ values FDR-corrected). The variable with the most differentially abundant taxa was constipation (Table 4). Of taxa previously associated with PD on the genus level, ${ }^{10}$ increased constipation severity was significantly associated with decreased abundance of Faecalibacterium $(p=0.022)$ and Roseburia $(p=0.008)$, physical exhaustion with a decrease in Bifidobacterium $(p=0.039)$, and possible RBD with a decrease in Lactobacillus $(p=0.023)$. Further, motor deficits indicating subthreshold parkinsonism were associated with a decrease in Odoribacter $(p=0.031)$. Possible RBD was further associated with a decrease in Faecalicoccus $(p=0.017)$ and Victivallis $(p=0.017)$, and an increase in the abundance of Haemophilus ( $p=0.003$ ). Urate-lowering medication was associated with higher abundance of Clostridium III $(p=0.005)$ and Parasutterella $(p=0.032)$. The taxa Prevotella and Akkermansia were not statistically significant in any of the differential abundance comparisons.

\section{Discussion}

The present study investigated associations between gut microbial composition, risk markers and prodromal markers of PD, subthreshold parkinsonism, and a wide range of potential confounders in 666 elderly individuals. Among these markers, particularly physical activity, constipation, possible RBD, smoking, and subthreshold parkinsonism were associated with alterations in microbial community composition. Moreover, age, sex, occupational solvent exposure, and an interaction of subthreshold parkinsonism and physical activity were associated with different microbial measures. As expected, effect sizes of individual markers and factors ( $\leq 1 \%$ explained variance) and multifactorial models $(\sim 8 \%)$ were small. ${ }^{16,17}$ None of the microbial measures was associated with substantia nigra hyperechogenicity, olfactory loss, depression, orthostatic hypotension, urinary/erectile dysfunction, family history of $\mathrm{PD}$, or the overall prodromal $\mathrm{PD}$ probability calculated based on marker profiles of individuals.

The relative abundances of bacterial taxa were mostly similar to other samples of healthy elderly individuals. ${ }^{32}$ Lower relative abundance of, for example, Bacteroides compared to previous findings may be explained by differences in cohort composition due to recruitment, demographic, regional, and/or lifestyle factors.

Age, constipation, and low physical activity were associated with increasing and occupational solvent exposure with decreasing within-sample $\alpha$-diversity. Higher $\alpha$-diversity in PD patients compared to controls has been reported, ${ }^{33-35}$ yet overall evidence is inconsistent, ${ }^{10}$ and it is unclear whether $\alpha$-diversity and PD are linked. Similar to our results, older age in an elderly population has been associated with higher $\alpha$-diversity. ${ }^{36}$ However, statistical modeling of such effects is complicated by the diseases, medications, and lifestyle changes often associated with advanced age. Regarding constipation, higher $\alpha$-diversity has been linked to harder stool consistency ${ }^{16}$ and functional constipation. ${ }^{37}$ Autonomous dysfunction related to prodromal PD may contribute to these $\alpha$-diversity effects, which however need to be disentangled from other potentially important factors, such as diet, stool water content and transit time, and bacterial growth rates. ${ }^{38}$ Physical inactivity increases the risk of many chronic diseases ${ }^{20}$ including $\mathrm{PD}^{15}$; conversely, being active may lead to lower prevalence of prodromal PD markers such as 
TABLE 3. Associations of Enterotypes with Risk and Prodromal Markers of Parkinson Disease and Additional Factors

\begin{tabular}{|c|c|c|c|c|c|c|c|c|c|}
\hline & \multicolumn{3}{|c|}{$\begin{array}{l}\text { Bacteroides vs } \\
\text { Firmicutes }\end{array}$} & \multicolumn{3}{|c|}{$\begin{array}{l}\text { Bacteroides vs } \\
\text { Prevotella }\end{array}$} & \multicolumn{3}{|c|}{$\begin{array}{l}\text { Firmicutes vs } \\
\text { Prevotella }\end{array}$} \\
\hline & Estimate & SE & $p$ & Estimate & SE & $p$ & Estimate & SE & $p$ \\
\hline Intercept & -1.44 & 0.44 & $0.00092^{\mathrm{a}}$ & -0.28 & 0.26 & 0.28121 & 1.17 & 0.47 & $0.01299^{\mathrm{b}}$ \\
\hline \multicolumn{10}{|l|}{ Risk markers } \\
\hline Physical activity (h sport/wk) & -0.58 & 0.16 & $0.00031^{\mathrm{a}}$ & -0.25 & 0.09 & $0.00313^{\mathrm{b}}$ & 0.33 & 0.17 & 0.05763 \\
\hline Male sex (no, yes) & -0.60 & 0.34 & 0.08162 & 0.25 & 0.20 & 0.22016 & 0.85 & 0.38 & $0.02355^{\mathrm{b}}$ \\
\hline \multicolumn{10}{|l|}{ Prodromal markers } \\
\hline $\begin{array}{l}\text { Constipation (sum score of Rome III } \\
\text { criteria items of functional constipation) }\end{array}$ & 0.07 & 0.03 & $0.02404^{\mathrm{b}}$ & -0.07 & 0.03 & $0.04502^{\mathrm{b}}$ & -0.14 & 0.04 & $0.00133^{\mathrm{b}}$ \\
\hline $\begin{array}{l}\text { Subthreshold parkinsonism } \\
\text { (no, borderline, yes) }\end{array}$ & 0.43 & 0.30 & 0.14722 & -0.36 & 0.30 & 0.23427 & -0.79 & 0.40 & $0.04628^{\mathrm{b}}$ \\
\hline \multicolumn{10}{|l|}{ Additional adjustment factors } \\
\hline Legumes (score) & -1.08 & 0.36 & $0.00285^{b}$ & 0.07 & 0.18 & 0.67751 & 1.16 & 0.39 & $0.00274^{\mathrm{b}}$ \\
\hline Functional bloating (no, yes) & 0.95 & 0.45 & $0.03235^{\mathrm{b}}$ & -0.59 & 0.46 & 0.20614 & -1.54 & 0.59 & $0.00906^{\mathrm{b}}$ \\
\hline Vegetarian (no, yes) & 1.05 & 0.51 & $0.04091^{\mathrm{b}}$ & 0.38 & 0.57 & 0.50650 & -0.68 & 0.65 & 0.29823 \\
\hline \multicolumn{10}{|c|}{$\begin{array}{l}\text { a Significant effects of risk and prodromal markers in logistic regressions after Bonferroni correction for multiple testing (also considering } 3 \text { group com- } \\
\text { parisons, } p<0.0009 \text { ). } \\
{ }^{b} \text { Effects with an uncorrected } p<0.05 \text {. } \\
\text { SE = standard error. }\end{array}$} \\
\hline
\end{tabular}

constipation. ${ }^{39}$ The effects of inactivity could be related to the processes observed in PD, that is, increased colonic inflammation, ${ }^{7}$ immune responses, ${ }^{6}$ and intestinal barrier permeability. ${ }^{5}$ Exhaustion from climbing stairs, an indicator of low cardiorespiratory fitness, was associated with a reduction in $\alpha$-diversity, in line with earlier studies indicating lower diversity in lower fitness ${ }^{40}$ and frailty. ${ }^{41}$ This apparently contradictory result may be partly explained by differences between physical activity and capability. Moreover, the PD risk marker occupational solvent exposure ${ }^{15}$ was associated with a decrease in $\alpha$-diversity. Strengths and directions of effects of different risk and prodromal markers on microbial composition may vary based on the underlying pathophysiological pathways, potentially explaining why the overall prodromal PD probability calculated based on comprehensive marker profiles showed no significant association with $\alpha$-diversity.

$\beta$-Diversity (between samples) has been consistently shown to differ between PD patients and controls. ${ }^{10}$ Several PD risk and prodromal markers were associated with $\beta$-diversity even in multifactorial models while considering potential confounders. These findings suggest that microbial composition might already be altered in the prodromal phase of PD. Although $\alpha$ - and $\beta$-diversity are not directly related, constipation and physical inactivity were again among the variables explaining most of the variance. While showing a nonsignificant interaction effect for $\alpha$-diversity $(p=.088)$, physical activity and motor deficits indicating subthreshold parkinsonism showed a significant interaction effect for $\beta$-diversity, and thus betweensample dissimilarities in microbial composition depend on the interaction of both markers. It remains speculative to what degree these associations play a specific role in prodromal PD. Links between $\beta$-diversity and possible RBD, sex, and smoking further support the concept of microbial changes preceding PD. While replicating effects of antidiabetic and beta-blocker medication, ${ }^{17}$ our study showed the most consistent associations of $\alpha$ - and $\beta$-diversity and differential abundance for urate-lowering medication. Urate is a powerful antioxidant linked to reduced PD risk, ${ }^{14}$ and our results may implicate gut microbiota in this context. Dark bread was the only dietary factor associated with $\beta$-diversity. Dark bread can be seen as an indicator of high fiber consumption, and thus presumably better intestinal barrier integrity and short-chain fatty acid (SCFA) production. ${ }^{42}$ Because both have been suggested 

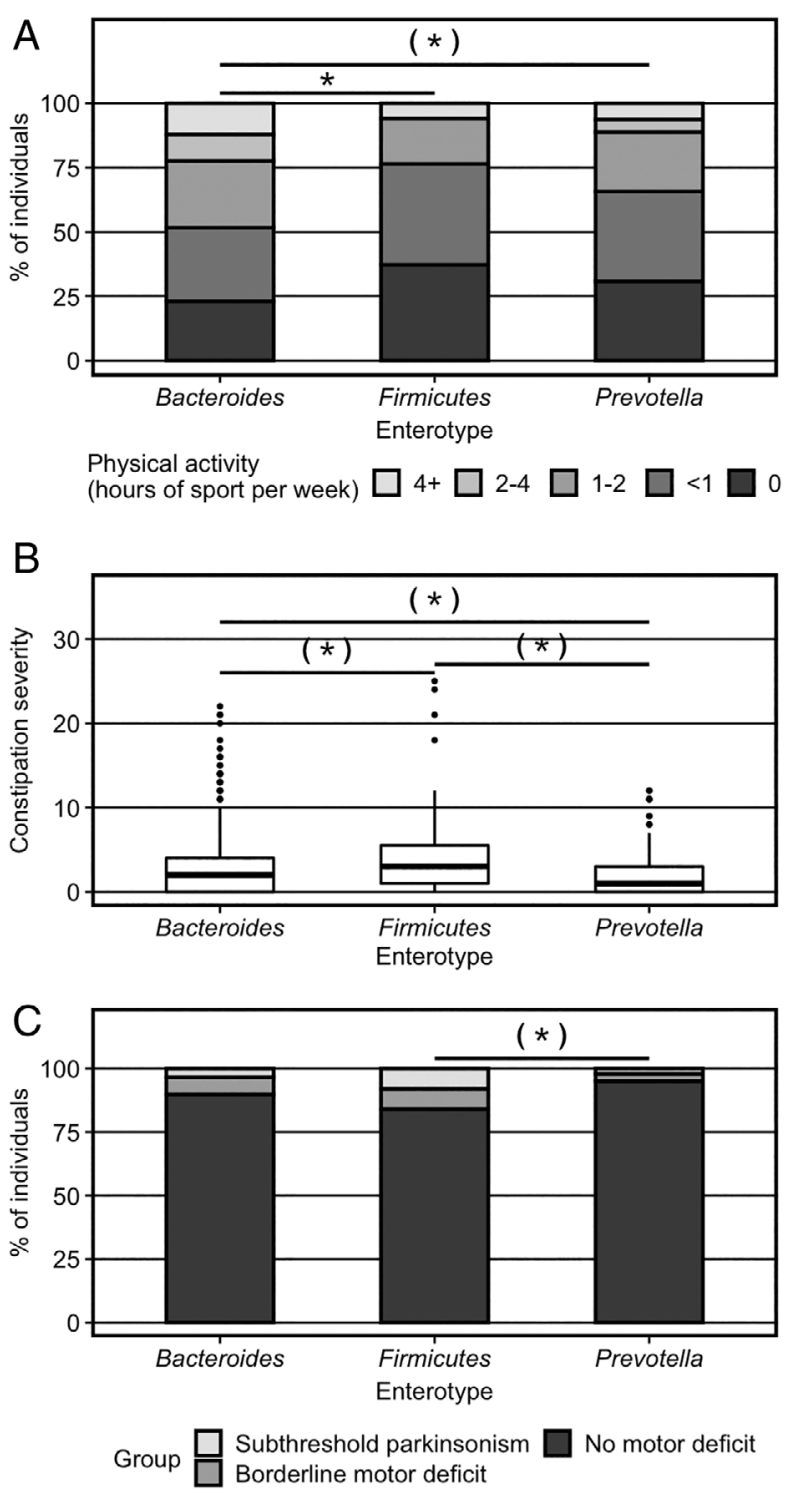

FIGURE 4: Enterotype group differences regarding (A) proportions of levels of physical activity, (B) severity of constipation, and (C) proportions of individuals with motor deficits indicating subthreshold parkinsonism. *Significant after Bonferroni correction ( $p<0.0009)$; $(*)$ indicates effects with an uncorrected $p<0.05$.

to be impaired in $\mathrm{PD}^{5,43}$ and to play a role in several processes along the microbiota-gut-brain axis, ${ }^{42}$ dietary factors might also be important in prodromal PD. After physical activity, BMI explained the most variance in $\beta$-diversity. For some dietary factors linked to BMI, independent effects might have been too small to reach the threshold for statistical significance.

Subthreshold parkinsonism was least frequently present in individuals with the Prevotella-enriched compared to the Firmicutes-enriched enterotype. This finding is in line with the reduced abundance of Prevotella in PD, ${ }^{10}$ in more severely progressing $\mathrm{PD},{ }^{11}$ and in $\mathrm{RBD}^{12}$ compared to controls. Thus, the present study supports the relevance of Prevotella in prodromal PD. Microbial changes due to constipation are often argued to confound microbiome analysis of PD patients. The Prevotella-enriched enterotype was also the least common in individuals with high constipation severity scores. Subjects with high scores typically had the Firmicutes-enriched enterotype in accordance with previous findings. ${ }^{44}$ Viewing constipation in PD as being linked to a disturbed enteric nervous system showing similar cellular changes to those of affected brain structures may suggest common relevance of Prevotella for prodromal dysautonomic and motor deficits. In this light, constipation may not be confounding, but may reflect a common pathogenic process. Although constipation may exert effects on microbial composition via several plausible mechanisms, such effects may be different in diseased individuals as compared to healthy subjects, as recently demonstrated in PD for the association between $\alpha$-diversity and stool consistency. ${ }^{11}$ Physical inactivity was more frequently observed in individuals with the Firmicutesenriched enterotype, whereas the Bacteroides-enriched enterotype was more frequent in active subjects. Although consistent with some evidence gained from human ${ }^{45}$ and mouse studies, ${ }^{46}$ processes underlying the links between physical activity, other prodromal markers in $\mathrm{PD},{ }^{39}$ obesity, nutrition, and microbial composition are complex and need to be further investigated.

The results of differential abundance analyses were partly consistent with previous findings, but some taxa reported for $\mathrm{PD}^{10}$ and $\mathrm{RBD}^{12}$ showed no association with risk and prodromal markers. In contrast with enterotype analyses, no significant differential abundance effect was observed for Prevotella, which may be partly explained by differences in covariates considered. Among other candidate genera, Akkermansia showed no significant association with any of the risk and prodromal markers. However, possible RBD showed several differentially abundant taxa, which have not been previously associated with $\mathrm{PD}^{10}$ or $\mathrm{RBD}^{12}$ (Faecalicoccus, Victivallis, and Haemophilus). Lactobacillus was decreased in individuals with possible RBD, whereas an increase in PD patients has previously been shown repeatedly. ${ }^{10}$ It is possible that subtypes of prodromal PD exist with varying involvement of the gut (eg, RBD representing a subtype with early autonomic denervation). ${ }^{47}$ Also, the microbiome may (differentially) change over time, that is, from prodromal to clinically established PD. Such complexity may hamper the identification of (prodromal) PD-related microbiome signatures. However, these signatures may allow for early stratification of individuals based on their microbiome (and underlying or inherent pathologies), and early and targeted therapeutic interventions. 


\begin{tabular}{|c|c|c|c|}
\hline & Family Level & Genus Level & OTU Level \\
\hline \multicolumn{4}{|l|}{ Risk markers } \\
\hline Physical activity (h sport/wk) & 4 & 5 & 7 \\
\hline Smoking (pack-years) & 1 & 1 & 12 \\
\hline \multicolumn{4}{|l|}{ Prodromal markers } \\
\hline $\begin{array}{l}\text { Constipation (sum score of Rome III criteria } \\
\text { items of functional constipation) }\end{array}$ & 10 & 25 & 51 \\
\hline Possible RBD (no, yes) & 4 & 4 & 4 \\
\hline Subthreshold parkinsonism (no, borderline, yes) & 0 & 1 & 4 \\
\hline \multicolumn{4}{|l|}{ Additional adjustment factors } \\
\hline Age (yr) & 0 & 2 & 5 \\
\hline Exhaustion from climbing stairs (no, yes) & 3 & 5 & 9 \\
\hline Urate-lowering medication (no, yes) & 3 & 2 & 5 \\
\hline Antihypertensive medication (no, yes) & 1 & 1 & 1 \\
\hline Diabetes medication (no, yes) & 2 & 5 & 9 \\
\hline
\end{tabular}

Constipation severity was significantly associated with decreased abundance of Faecalibacterium and Blautia, SCFA-producing taxa that can exert positive effects on the intestinal mucosa ${ }^{48}$ and are decreased in PD. ${ }^{10}$ The finding of a decrease in Odoribacter in individuals with subthreshold parkinsonism has potential relevance for prodromal PD. Odoribacter is a taxon involved in SCFA production and tryptophan metabolism. It might be relevant for gastrointestinal integrity and serotonergic bowel dysfunction (prolonged transit time) as well as central nervous dysfunction (anxiety) as suggested by findings in an autism mouse model. ${ }^{49}$

The present study has several limitations. (1) Some markers, and dietary and medication data, were assessed using self-reports. Although those assessments were structured and highly standardized, quantitative measures or medical records might be more accurate, for example, for assessing physical activity, diets and medication. (2) Interaction analyses were limited to those involving physical activity, given its relevance as a PD risk marker ${ }^{15}$ and for prodromal PD markers. ${ }^{39}$ Further research on marker interactions and clusters as well as factors underlying potential biases is needed to better model the complexity of microbial associations. (3) Stool samples were not frozen immediately after defecation but after postal delivery, and the delay may constitute a technical confounder. However, collection tubes contained a DNA stabilizer, and the impact of delayed freezing on microbial composition should therefore be minimal. ${ }^{11,50}$ (4) Some risk and prodromal markers were present at lower frequency, and lower statistical power might have contributed to some non-significant findings.

In conclusion, several risk and prodromal markers in $\mathrm{PD}$, in particular markers related to motor aspects and constipation, were associated with altered microbial $\alpha$ and $\beta$-diversity, enterotypes, and bacterial abundance. Constipation, physical activity, possible RBD, urate levels, smoking, and subthreshold parkinsonism might be particularly linked to the prodromal microbiome in PD. However, many other markers predictive of $\mathrm{PD}$ and overall prodromal PD probability values showed no significant association with any microbial measure. Prodromal microbial changes might only be observable in subgroups with specific marker constellations. The functional roles of these markers and associated microbiota for intestinal permeability, stool immune mediators, colonic inflammation, and the systemic interactions with the host organism need further investigation. The prodromal microbiome(s) in $\mathrm{PD}$, temporal dynamics of microbiota toward PD diagnosis, and etiological relevance in prodromal PD should be 
investigated based on prospective marker profiles and (multi)omics data in incident PD cases.

\section{Acknowledgment}

Samples were obtained from the Neuro-Biobank of the University of Tübingen, Germany (https://www.hihtuebingen.de/en/about-us/core-facilities/biobank/). This biobank is supported by the local university, the Hertie Institute for Clinical Brain Research, and the German Center for Neurodegenerative Diseases (DZNE). The TREND study is being conducted at the University Hospital Tübingen and has been supported by the Hertie Institute for Clinical Brain Research, the DZNE, the Geriatric Center of Tübingen, the Center for Integrative Neuroscience, Teva Pharmaceutical Industries, Union Chimique Belge, Janssen Pharmaceuticals, and the International Parkinson Foundation. This study was funded further by the Academy of Finland $(295724,310835)$ and the Finnish Medical Foundation. The supporting institutions had no influence on the design, conduct, or analysis of the study.

We thank the participants for their continued participation in the TREND study and for providing their stool samples; the numerous (doctoral) students and study nurses, who actively contributed to study organization, and data collection, entry, and monitoring; Dr A. Caliebe for statistical consultancy advice; and A. Gashi, E. Mustanoja, and P. Collin for their work on biological sample processing at the DNA Sequencing Lab.

\section{Author Contributions}

S.H.e., K.B., G.W.E., W.M., D.B., P.A., and F.S. contributed to the conception and design of the study; S.H. e., S.H.a., V.T.E.A., L.P., C.D., C.S., K.B., U.S., A.-K.v. T., L.P., P.A., and F.S. contributed to the acquisition and analysis of data; S.H.e., V.T.E.A., W.M., P.A., and F.S. contributed to drafting the text and preparing the figures.

The TREND organization team consists of Corina Maetzler, Susanne Nußbaum, Dr Anna-Katharina von Thaler, Ulrike Sunkel, Christian Mychajliw, and Ramona Täglich.

\section{Data Availability}

TREND data are available upon request. Microbiome sequence raw data are available under the European Nucleotide Archive accession number PRJEB32920.

\section{Potential Conflicts of Interest}

V.T.E.A., L.P., P.A., and F.S. are inventors on granted patents related to the use of microbiota analysis in the diagnosis of PD (FI127671B, US10139408B2) and pending patents related to the use of microbiota in the diagnosis and treatment of PD (US16/186,663, EP3149205). These patents/patent applications are currently assigned to NeuroInnovation $\mathrm{Oy}$ and licensed to NeuroBiome. NeuroInnovation Oy provides clinical neurological services for patients and health care providers as well as consultant services in the field of neurology and microbiota. NeuroBiome pursues the development and commercialization of diagnostic and therapeutic applications of microbiota for PD. Currently, no such products are marketed by NeuroBiome or NeuroInnovation Oy. F.S. owns $85 \%$ of shares of NeuroInnovation $\mathrm{Oy}$ and $100 \%$ of shares of NeuroBiome. None of the abovementioned inventors has received any fees or royalties from these companies related to microbiota-related products, but they may do so in the future if development and commercialization are successful. F.S. is a member of the scientific advisory board of and has received fees and stock options from Axial Biotherapeutics, a company that is developing gut-brain axis-related therapeutics for PD and autism spectrum disorder.

\section{References}

1. Stokholm MG, Danielsen EH, Hamilton-Dutoit SJ, Borghammer P. Pathological $\alpha$-synuclein in gastrointestinal tissues from prodromal Parkinson disease patients. Ann Neurol 2016;79:940-949.

2. Schrag A, Horsfall L, Walters K, et al. Prediagnostic presentations of Parkinson's disease in primary care: a case-control study. Lancet Neurol 2015;14:57-64.

3. Abbott RD, Petrovitch $H$, White LR, et al. Frequency of bowel movements and the future risk of Parkinson's disease. Neurology 2001;57: $456-462$.

4. Breen DP, Halliday GM, Lang AE. Gut-brain axis and the spread of $\alpha$-synuclein pathology: vagal highway or dead end? Mov Disord 2019;34:307-316.

5. Forsyth CB, Shannon KM, Kordower JH, et al. Increased intestinal permeability correlates with sigmoid mucosa alpha-synuclein staining and endotoxin exposure markers in early Parkinson's disease. PLoS One 2011;6:e28032.

6. Houser MC, Chang J, Factor SA, et al. Stool immune profiles evince gastrointestinal inflammation in Parkinson's disease. Mov Disord 2018;33:793-804.

7. Devos D, Lebouvier T, Lardeux B, et al. Colonic inflammation in Parkinson's disease. Neurobiol Dis 2013;50:42-48.

8. Sampson TR, Debelius JW, Thron T, et al. Gut microbiota regulate motor deficits and Neuroinflammation in a model of Parkinson's disease. Cell 2016;167:1469-1480. e12.

9. Scheperjans F, Aho V, Pereira PAB, et al. Gut microbiota are related to Parkinson's disease and clinical phenotype. Mov Disord 2015;30: 350-358

10. Boertien JM, Pereira PAB, Aho VTE, Scheperjans F. Increasing comparability and utility of gut microbiome studies in Parkinson's disease: a systematic review. J Parkinsons Dis 2019;9:S297-S312. 
11. Aho VTE, Pereira PAB, Voutilainen $S$, et al. Gut microbiota in Parkinson's disease: temporal stability and relations to disease progression. EBioMedicine 2019;44:691-707.

12. Heintz-Buschart A, Pandey $U$, Wicke $T$, et al. The nasal and gut microbiome in Parkinson's disease and idiopathic rapid eye movement sleep behavior disorder. Mov Disord 2018;33:88-98.

13. Mahlknecht P, Seppi K, Poewe W. The concept of prodromal Parkinson's disease. J Parkinsons Dis 2015;5:681-697.

14. Berg D, Postuma RB, Adler $\mathrm{CH}$, et al. MDS research criteria for prodromal Parkinson's disease. Mov Disord 2015;30:1600-1611.

15. Heinzel S, Berg D, Gasser T, et al. Update of the MDS research criteria for prodromal Parkinson's disease. Mov Disord 2019;34: 1464-1470.

16. Falony $\mathrm{G}$, Joossens $\mathrm{M}$, Vieira-Silva $\mathrm{S}$, et al. Population-level analysis of gut microbiome variation. Science 2016;352:560-564

17. Zhernakova A, Kurilshikov A, Bonder MJ, et al. Population-based metagenomics analysis reveals markers for gut microbiome composition and diversity. Science 2016;352:565-569.

18. Forslund K, Hildebrand F, Nielsen T, et al. Disentangling type 2 diabetes and metformin treatment signatures in the human gut microbiota. Nature 2015;528:262-266.

19. Valles-Colomer M, Falony G, Darzi Y, et al. The neuroactive potential of the human gut microbiota in quality of life and depression. Nat Microbiol 2019:4:623-632.

20. Booth FW, Roberts CK, Thyfault JP, et al. Role of inactivity in chronic diseases: evolutionary insight and pathophysiological mechanisms. Physiol Rev 2017;97:1351-1402

21. Langsetmo $L$, Johnson $A$, Demmer $R T$, et al. The association between objectively measured physical activity and the gut microbiome among older community dwelling men. J Nutr Heal Aging 2019:23:538-546.

22. Codella R, Luzi L, Terruzzi I. Exercise has the guts: how physical activity may positively modulate gut microbiota in chronic and immunebased diseases. Dig Liver Dis 2018;50:331-341.

23. Longstreth GF, Thompson WG, Chey WD, et al. Functional bowel disorders. Gastroenterology 2006;130:1480-1491.

24. Pilotto A, Heinzel S, Suenkel U, et al. Application of the movement disorder society prodromal Parkinson's disease research criteria in 2 independent prospective cohorts. Mov Disord 2017;32:1025-1034.

25. Goetz CG, Tilley BC, Shaftman SR, et al. Movement Disorder Society-sponsored revision of the unified Parkinson's disease rating scale (MDS-UPDRS): scale presentation and clinimetric testing results. Mov Disord 2008:23:2129-2170.

26. Somervuo $\mathrm{P}$, Koskinen $\mathrm{P}$, Mei $\mathrm{P}$, et al. BARCOSEL: a tool for selecting an optimal barcode set for high-throughput sequencing. BMC Bioinformatics 2018;19:257.

27. Martin M. Cutadapt removes adapter sequences from high-throughput sequencing reads. EMBnetjournal 2011;17:10-12.

28. Schloss PD, Westcott SL, Ryabin T, et al. Introducing mothur: opensource, platform-independent, community-supported software for describing and comparing microbial communities. Appl Environ Microbiol 2009;75:7537-7541.

29. Kozich JJ, Westcott SL, Baxter NT, et al. Development of a dualindex sequencing strategy and curation pipeline for analyzing amplicon sequence data on the miseq illumina sequencing platform. Appl Environ Microbiol 2013;79:5112-5120

30. McMurdie PJ, Holmes S. Phyloseq: an R package for reproducible interactive analysis and graphics of microbiome census data. PLoS One 2013;8:e61217.
31. Le Chatelier $E$ et al. Richness of human gut microbiome correlates with metabolic markers. Nature 2013;500:541-546.

32. Claesson MJ, Cusack S, O'Sullivan O, et al. Composition, variability, and temporal stability of the intestinal microbiota of the elderly. Proc Natl Acad Sci U S A 2011;108:4586-4591.

33. Barichella M, Severgnini M, Cilia R, et al. Unraveling gut microbiota in Parkinson's disease and atypical parkinsonism. Mov Disord 2019; 34:396-405.

34. Qian $Y$, Yang $X, X u$ S, et al. Alteration of the fecal microbiota in Chinese patients with Parkinson's disease. Brain Behav Immun 2018;70: 194-202.

35. Keshavarzian A, Green SJ, Engen PA, et al. Colonic bacterial composition in Parkinson's disease. Mov Disord 2015:30:1351-1360.

36. Odamaki T, Kato K, Sugahara H, et al. Age-related changes in gut microbiota composition from newborn to centenarian: a cross-sectional study. BMC Microbiol 2016;16:90

37. Mancabelli L, Milani C, Lugli GA, et al. Unveiling the gut microbiota composition and functionality associated with constipation through metagenomic analyses. Sci Rep 2017;7:9879.

38. Vandeputte D, Falony G, Vieira-Silva S, et al. Stool consistency is strongly associated with gut microbiota richness and composition, enterotypes and bacterial growth rates. Gut 2016;65:57-62.

39. Hughes KC, Gao X, Molsberry S, et al. Physical activity and prodromal features of Parkinson disease. Neurology 2019;93:e2157-e2169.

40. Estaki M, Pither J, Baumeister $P$, et al. Cardiorespiratory fitness as a predictor of intestinal microbial diversity and distinct metagenomic functions. Microbiome 2016:4:42.

41. Jackson MA, Jeffery IB, Beaumont $M$, et al. Signatures of early frailty in the gut microbiota. Genome Med 2016;8:8.

42. Dalile B, Van Oudenhove L, Vervliet B, Verbeke K. The role of shortchain fatty acids in microbiota-gut-brain communication. Nat Rev Gastroenterol Hepatol 2019;16:461-478.

43. Unger MM, Spiegel J, Dillmann KU, et al. Short chain fatty acids and gut microbiota differ between patients with Parkinson's disease and age-matched controls. Parkinsonism Relat Disord 2016;32:66-72.

44. Zhu L, Liu W, Alkhouri R, et al. Structural changes in the gut microbiome of constipated patients. Physiol Genomics 2014;46:679-686.

45. Morita $E$, Yokoyama $H$, Imai $D$, et al. Aerobic exercise training with brisk walking increases intestinal bacteroides in healthy elderly women. Nutrients 2019;11:868

46. Ticinesi A, Lauretani F, Tana C, et al. Exercise and immune system as modulators of intestinal microbiome: implications for the gut-muscle axis hypothesis. Exerc Immunol Rev 2019;25:84-95.

47. Borghammer $P$, Van Den Berge N. Brain-first versus gut-first Parkinson's disease: a hypothesis. J Parkinsons Dis 2019;9:S281S295.

48. Venegas DP, De la Fuente MK, Landskron G, et al. Short chain fatty acids (SCFAs)-mediated gut epithelial and immune regulation and its relevance for inflammatory bowel diseases. Front Immunol 2019; $10 \cdot 277$

49. Golubeva AV, Joyce SA, Moloney G, et al. Microbiota-related changes in bile acid \& tryptophan metabolism are associated with gastrointestinal dysfunction in a mouse model of autism. EBioMedicine 2017;24:166-178.

50. Ilett EE, Jørgensen M, Noguera-Julian M, et al. Gut microbiome comparability of fresh-frozen versus stabilized-frozen samples from hospitalized patients using 16S rRNA gene and shotgun metagenomic sequencing. Sci Rep 2019;9:13351. 